\title{
The influence of epidural blockade on gut permeability in patients undergoing open surgical repair of abdominal aortic aneurysm
}

\author{
Radosław Owczuk ${ }^{1}$, Anna Dylczyk-Sommer ${ }^{1}$, Jacek Wojciechowski², Monika Paszkiewicz ${ }^{3}$, \\ Maria Wujtewicz ${ }^{1}$, Piotr Stepnowski ${ }^{3}$, Paweł Twardowski ${ }^{1}$, Wioletta Sawicka', \\ Michał Domżalski ${ }^{1}$, Magdalena A. Wujtewicz ${ }^{4}$ \\ ${ }^{1}$ Department of Anaesthesiology and Intensive Therapy, Medical University of Gdańsk, Poland \\ ${ }^{2}$ Department of Cardiac and Vascular Surgery, Medical University of Gdańsk, Poland \\ ${ }^{3}$ Department of Environmental Analysis, Faculty of Chemistry, University of Gdańsk, Poland \\ ${ }^{4}$ Department of Ophtalmology, Medical University of Gdańsk, Poland
}

\begin{abstract}
Background: Abdominal aortic clamping during aneurysm repair may cause a decrease in splanchnic blood flow and deterioration of gut barrier integrity. Epidural blocks have beneficial effects on vital organs during abdominal surgery, but sparse data are available on the influence on gut permeability during open aortic surgery. The aim of this study was to verify the hypothesis that epidural blocks may have beneficial effects on intestine permeability changes. Methods: Seventy individuals undergoing elective open abdominal aortic aneurysm repair were randomly assigned to receive either balanced anaesthesia (continuous epidural and general anaesthesia, group E\&G) or only general anaesthesia (group G). For group E\&G, an epidural catheter was inserted into the epidural space before the induction of general anaesthesia. Ropivacaine was used for intraoperative and postoperative blocks. For both groups general anaesthesia was maintained with sevoflurane. For group $G$, analgesia was provided with remifentanil. The assessments of gut function were based on measurements of the absorption and percentages of urinary excretion of four sugars (m 3-O-methyl-D-glucose, D-xylose, L-rhamnose and lactulose) and the lactulose/rhamnose (L/R) ratio.

Results: No intergroup differences were observed for sugar recovery or L/R ratio. Significant decreases in 3-O-methylD-glucose, D-xylose, and L-rhamnose recoveries were revealed in both examined groups when comparing the results obtained at 12 and 24 hours following the administration of anaesthesia. The rate of blood pressure decrease was significantly higher in group $E \& G$.

Conclusions: Aortic clamping during open abdominal aortic repair led to unfavorable changes in intestinal permeability. Epidural block did not attenuate this deterioration.
\end{abstract}

Key words: aortic aneurysm, repair, open surgery; gut, barrier, assessment; anaesthesia, regional, epidural blockade

Anaesthesiology Intensive Therapy 2016, vol. 48, no 2, 122-127

Traditional open surgery remains the most common method of repairing abdominal aortic aneurysms, although the use of the endovascular technique is clearly increasing [1-3]. Major open aortic surgery, particularly the repair of ruptured aneurysms, is associated with a relatively high rate of complications [4]. Unfortunately, complications have been reported during scheduled procedures even in well-prepared patients, which have occurred due to many factors. Aortic clamping may be responsible for many of these complications, including visceral ischemia. There are many reports of kidney injury following aortic clamping and its prophylaxis [5]. By contrast, sparse data are available regarding the influence of this procedure on gut blood flow and wall permeability. This procedure can have potentially dangerous consequences, such as toxin or bacterial translocation into the bloodstream. It has been 
shown that longer periods of visceral ischemia in patients undergoing supraceliac cross-clamping are associated with higher levels of intraoperative CD11b expression, severe sepsis, and organ failure [6].

Open aortic surgery is frequently performed under balanced general and epidural anaesthesia, and epidural block is suitable for postoperative pain treatment, although its popularity has decreased in recent years [7]. Thoracic epidural anaesthesia (TEA) reduces cardiac and splanchnic sympathetic activities, thereby positively influencing the perioperative functioning of vital organ systems. TEA appears to reduce the occurrence of gut injury during major surgery as long as its systemic hemodynamic effects are adequately controlled [8]. No data are available from human studies regarding the influence of epidural blocks on gut permeability changes induced by abdominal aortic clamping.

The aim of this study was to verify the hypothesis that epidural blocks may have beneficial effects on intestinal permeability changes resulting from open surgical repair of abdominal aortic aneurysm.

\section{METHODS}

After obtaining approval from the Bioethical Committee of the Medical University of Gdansk and written informed consent from the study participants, 70 individuals were recruited to take part in this study. Patients were of both sexes and between 40 and 75 years of age and were already scheduled to undergo elective open abdominal aortic aneurysm repair. Patients with chronic intestinal diseases (e.g., Crohn's disease or ulcerative colitis), malabsorption syndromes, or chronic kidney disease were excluded. Patients were also excluded if they had any contraindications for an epidural block or did not agree to receive this type of anaesthesia.

A randomization scheme was generated using the generator of Wichmann and Hill, as modified by McLeod (available at http://www.randomization.com). Patients were randomly allocated to receive either balanced anaesthesia (continuous epidural and general anaesthesia, group E\&G) or only general anaesthesia (group G). For group E\&G, an epidural catheter was inserted into the epidural space (T10-11 to L1-2 levels) before the induction of general anaesthesia. After confirmation of correct catheter placement (injection of a test dose of 3-4 $\mathrm{mL}$ of $2 \%$ lidocaine and 15 minutes of observation), $1 \%$ ropivacaine was injected through the catheter at a dose of $0.8-1.2 \mathrm{~mL}$ per anaesthetized segment, followed by continuous infusion of $6-12 \mathrm{~mL} \mathrm{~h}^{-1}$ of a $0.5 \%$ solution.

During the postoperative period, a $0.2 \%$ ropivacaine solution was used for epidural analgesia. The dose of local anaesthetic was adjusted individually according to the pain level of the patient. For group $E \& G$, general anaesthesia was maintained with $0.5-2.5$ vol\% of sevoflurane while the lungs were ventilated with a mixture of $33 \%$ oxygen and $67 \%$ nitrous oxide. Skeletal muscle relaxation was achieved with rocuronium bromide, using an intubating dose of $0.6 \mathrm{mg} \mathrm{kg}^{-1}$, followed by continuous infusion of $0.3-0.6 \mathrm{mg} \mathrm{kg}^{-1} \mathrm{hour}^{-1}$.

For group $\mathrm{G}$, analgesia was provided with remifentanil at an induction dose of $0.5-1 \mathrm{\mu g} \mathrm{kg}^{-1}$, followed by continuous infusion of $0.3-0.6 \mu \mathrm{g} \mathrm{kg}^{-1} \mathrm{~min}^{-1}$ during the maintenance phase. Similar to group E\&G, sevoflurane was used for the maintenance of general anaesthesia, and rocuronium bromide was used for muscle relaxation, and the same dosages were used for both groups.

Assessments of gut absorption and permeability were based on measurements of the absorption and percentages of urinary excretion of four sugars (mono- and disaccharides, including 3-O-methyl-D-glucose, D-xylose, L-rhamnose and lactulose).

A sugar solution containing $2 \mathrm{~g} \mathrm{~L}^{-1} 3-0$-methyl-D-glucose, $5 \mathrm{~g} \mathrm{~L}^{-1}$ of D-xylose, $10 \mathrm{~g} \mathrm{~L}^{-1}$ of L-rhamnose and $50 \mathrm{~g} \mathrm{~L}^{-1}$ of lactulose, with an approximate osmolarity of 240 milliosmoles $\mathrm{L}^{-1}$, was prepared by the hospital pharmacy on the day of anaesthesia administration. A dose of $2 \mathrm{~mL} \mathrm{~kg}^{-1}$ of this mixture was administered through a nasogastric tube right after induction of anaesthesia. The urinary concentrations of the aforementioned saccharides were measured at the three following time points: during general anaesthesia administration and at 12 and 24 hours after induction of anaesthesia. At these time points, 3-hour urine collections were initiated through a urinary catheter, and $20-\mathrm{mL}$ urine samples were obtained from these 3-hour samples. The samples were stored at $-85^{\circ} \mathrm{C}$ until further analysis.

Urine sugar concentrations were determined by gas chromatography (GC) and gas chromatography-mass spectrometry (GC/MS). Because sugars are highly polar and have low volatility, chemical derivatization was required before GC-MS analysis. Sugars were reduced with sodium borohydride $(5 \mathrm{mg})$ in water $(500 \mu \mathrm{L})$ for 12 hours at room temperature. Then, several drops of acetic acid were added to remove the excess borohydride. Next, acetic acid/methanol $1: 9(200 \mu \mathrm{L})$ was added, and the mixtures were dried under a stream of nitrogen to remove the boric acid. This procedure was performed three times with acetic acid/methanol $(250 \mu \mathrm{L})$. After drying under vacuum over phosphorus pentoxide, dry pyridine $(100 \mu \mathrm{L})$ and acetic anhydride $(200 \mu \mathrm{L})$ were added, and the samples were incubated at $120^{\circ} \mathrm{C}$ for 2 hours in a reacti-block. The reaction mixtures were dried under a gentle stream of nitrogen while adding toluene $(500 \mu \mathrm{L}$, twice). The samples were dissolved in $\mathrm{CH}_{2} \mathrm{Cl}_{2}$ and injected $(1 \mu \mathrm{L})$ into a gas chromatograph. The individual sugars in the test mixture showed the following constant response factors: 1.503 for 3-O-methyl-D-glucose, 0.844 for L-rhamnose, 1.196 for D-xylose (defined relative to D-ribose), and 0.750 
for lactulose (determined relative to an internal standard, trehalose). This developed method can be used for qualitative and quantitative analyses of mono- and disaccharides in urine samples. However, the time required for analysis of one sample is too long, which precludes its clinical routine use. To optimize the derivatization procedure, reactions of mono- and disaccharides with hydroxylamine chlorohydrate $(100 \mu \mathrm{l})$ in pyridine were also performed $\left(90^{\circ} \mathrm{C}\right.$ for $1 \mathrm{~h})$. The obtained oximes were then converted to silyl derivatives with $N$-trimethylsilylimidazole $\left(100 \mu \mathrm{L}, 90^{\circ} \mathrm{C}\right.$ for $\left.1 \mathrm{~h}\right)$. This method was rapid, sensitive, and easy to automate for the study of intestinal permeability in clinical practice. The validation parameters were acceptable for analytical work. Urine sample preparation procedure for analyses of monoand disaccharides was also developed. DEAE Sephadex A-25 (200 mg) and washed ion exchange resin Amberlite XAD-2 $(500 \mathrm{mg}$ ) were added to $10 \mathrm{ml}$ of each thawed urine specimen for desalting. The mixture was vortex-mixed for $10 \mathrm{~s}$ and centrifuged for $10 \mathrm{~min}$ at $4000 \mathrm{rpm}$. The resulting supernatant was filtered through $0.45-\mu \mathrm{m}$ (pore size) disposable syringe filters and lyophilized.

\section{STATISTICAL ANALYSIS}

A minimal sample size of 34 individuals per group was calculated assuming an alpha level of 0.05 and a beta level of 0.80 for the detection of a mean difference in the $L / R$ ratio of 0.02 , based on a study by Fujii et al. that reported a mean (SD) L/R ratio of 0.049 (0.008) [9].

Statistical analysis was performed using STATISTICA 10.0 PL software (Polish version) (StatSoft, Tulsa, OK, USA). The normality of the data distribution was verified using the Shapiro-Wilk W test, and the homogeneity of variance was verified using Levene's test. Comparisons were performed using two-sided analysis of variance (ANOVA) for repeated measurements with subsequent analysis of significant differences using a post hoc method (Tukey's honest significant difference (HSD) test) if appropriate or the chi-squared test (with the Yates correction if necessary). A $P<0.05$ was indicative of a significant difference.

\section{RESULTS}

Demographic data and comorbidities are presented in Table 1. There were no significant differences in mean age, gender, or body mass among the three groups. Similarly, there were no differences in American Society of Anesthesiology (ASA) pre-operative status, the prevalence of coronary artery syndrome or brain stroke in anamnesis. The prevalences of diabetes and arterial hypertension were higher in group $\mathrm{G}$ ( $P=0.04$ for both diseases).

The aortic disorders that prompted the surgical procedures and coexisting vascular pathologies are shown in
Table 1. Patient demographics. Median $\left(25^{\text {th }}-75^{\text {th }}\right.$ percentile) or $n(\%)$ is shown

\begin{tabular}{lcc}
\hline & $\begin{array}{c}\text { Group E\&G } \\
(\mathbf{n}=35)\end{array}$ & $\begin{array}{c}\text { Group G } \\
(\mathbf{n}=35)\end{array}$ \\
\hline Age (years) & $69(63-75)$ & $68(65-74)$ \\
Sex F : M & $4: 31$ & $9: 26$ \\
Body mass $(\mathrm{kg})$ & $78(65-88)$ & $78(70-86)$ \\
ASA status; II : III & $13: 22$ & $11: 24$ \\
Comorbidities & $23(65.7 \%)$ & $31(88.6 \%)$ \\
Arterial hypertension & $4(11.4 \%)$ & $12(34.3 \%)$ \\
Diabetes & $19(54.3 \%)$ & $18(51.4 \%)$ \\
Coronary disease & $2(5.7 \%)$ & $3(8.6 \%)$ \\
History of stroke & & \\
\hline
\end{tabular}

Table 2. Aortic disorders that led to surgical procedures

\begin{tabular}{lcc}
\hline & $\begin{array}{c}\text { Group E\&G } \\
(\mathbf{n = 3 5 )}\end{array}$ & $\begin{array}{c}\text { Group G } \\
(\mathbf{n = 3 5 )}\end{array}$ \\
\hline Aortic aneurysm & $24(68.6 \%)$ & $30(85.7 \%)$ \\
$\begin{array}{l}\text { Aortic aneurysm coexisting with } \\
\text { aneurysm of one or both iliac arteries }\end{array}$ & $10(28.6 \%)$ & $4(11.4 \%)$ \\
$\begin{array}{l}\text { Aortic aneurysm coexisting with } \\
\text { stenosis of the iliac artery }\end{array}$ & $1(2.8 \%)$ & 0 \\
$\begin{array}{l}\text { Aortic aneurysm coexisting with } \\
\text { stenosis of the renal artery }\end{array}$ & 0 & $1(2.8 \%)$ \\
\hline
\end{tabular}

Table 3. Type of surgical procedure and duration of aortic cross-clamping. Median $\left(25^{\text {th }}-75^{\text {th }}\right.$ percentile) or $\mathrm{n}(\%)$ is shown.

\begin{tabular}{lccc}
\hline & $\begin{array}{c}\text { Group E\&G } \\
(\mathbf{n}=35)\end{array}$ & $\begin{array}{c}\text { Group G } \\
(\mathbf{n}=35)\end{array}$ & Pvalue \\
\hline Aortoaortic bypass & $19(54.3 \%)$ & $27(77.1 \%)$ & 0.16 \\
ABI & $3(8.6 \%)$ & $3(8.6 \%)$ & \\
ABF & $9(25.7 \%)$ & $4(11.4 \%)$ & \\
Ao-MI/MF & $4(11.4 \%)$ & $1(2.8 \%)$ & \\
Cross-clamping (min) & $40(31-52)$ & $35(28-44)$ & 0.14 \\
\hline
\end{tabular}

$\mathrm{ABI}$ - aortobiiliac bypass; ABF — aortobifemoral bypass; Ao-MI/MI - aortic bypass with one distal end in the iliac artery on one side and with the second distal end in the femoral artery on the other side of the body

Table 2. The type of surgical procedure and the duration of aortic cross-clamping are shown in Table 3. There were no significant differences among the types of procedures and the durations of aortic cross-clamping.

Intra- and postoperative events that could have influenced the results of the current study are shown in Table 4. Statistical analysis showed significant differences in the number of episodes of blood pressure drops between the groups $(P=0.008)$. There was no difference in the necessity for catecholamines during anaesthesia. The only catecho- 
Table 4. Intra- and postoperative events with potential influences on the study results

\begin{tabular}{|c|c|c|c|}
\hline & $\begin{array}{l}\text { Group E\&G } \\
(n=35)\end{array}$ & $\begin{array}{l}\text { Group G } \\
(n=35)\end{array}$ & $\begin{array}{c}P \\
\text { value }\end{array}$ \\
\hline $\begin{array}{l}\text { Significant blood pressure } \\
\text { decrease* }^{*}\end{array}$ & $22(62.8 \%)$ & 12 (34.3\%) & 0.008 \\
\hline Need for catecholamines & $8(22.9 \%)$ & $3(8.6 \%)$ & 0.09 \\
\hline $\begin{array}{l}\text { Intestines removed from } \\
\text { peritoneal cavity }\end{array}$ & $5(14.3 \%)$ & $1(2.8 \%)$ & 0.09 \\
\hline $\begin{array}{l}\text { Cross-clamping above } \\
\text { renal arteries }\end{array}$ & $1(2.8 \%)$ & $1(2.8 \%)$ & 1.0 \\
\hline Postoperative AKI & $2(5.7 \%)$ & $1(2.8 \%)$ & 1.0 \\
\hline
\end{tabular}

AKI - acute kidney injury; ${ }^{*}$ A significant blood pressure decrease was defined as a decrease in systolic pressure to below $90 \mathrm{~mm} \mathrm{Hg}$ that lasted for more than 15 minutes

Table 5. Sugar recoveries and lactulose/rhamnose (L/R) ratios for the studied groups, expressed as percentages of the intragastrically administered dose. Data are reported as medians $\left(25^{\text {th }}-75^{\text {th }}\right.$ percentiles). +12 and +24 hours indicate time after anaesthesia induction

\begin{tabular}{lll}
\hline & Group E\&G & Group G \\
\hline 3-O-methyl-D-glucose & & \\
\hline+12 hours & $4.55(2.28-5.43)$ & $5.09(1.03-5.56)$ \\
+24 hours & $0.95(0.60-1.61)^{*}$ & $0.62(0.59-1.75)^{*}$ \\
\hline D-xylose & & \\
\hline+12 hours & $5.24(4.76-6.62)$ & $5.09(4.10-5.47)$ \\
+24 hours & $2.39(1.93-2.72)^{*}$ & $2.54(0.45-2.79)^{*}$ \\
\hline L-rhamnose & & \\
\hline+12 hours & $1.09(0.97-1.21)$ & $1.18(0.98-1.31)$ \\
+24 hours & $0.34(0.25-0.56)^{*}$ & $0.32(0.20-0.72)^{*}$ \\
\hline Lactulose & & \\
\hline+12 hours & $0.36(0.13-0.57)$ & $0.28(0.12-0.48)$ \\
+24 hours & $0.23(0.11-0.25)$ & $0.24(0.09-0.45)$ \\
\hline L/R ratio & & \\
\hline+12 hours & $0.18(0.11-0.56)$ & $0.21(0.10-0.43)$ \\
+24 hours & $0.22(0.15-0.64)$ & $0.28(0.17-0.65)$ \\
\hline
\end{tabular}

* $P<0.05$ compared with the value at the $12^{\text {th }}$ hour in the same group

lamine used was dopamine, the dose range of which was between 5 and $10 \mu \mathrm{g} \mathrm{kg}^{-1} \mathrm{~min}^{-1}$.

In both examined groups, traces of 3-O-methyl-D-glucose, D-xylose, and L-rhamnose were detected in the urine samples collected during the first three hours of anaesthesia from 3 individuals in group E\&G and 3 individuals from group G. No subjects exhibited detectable lactulose in their urine, which is why the lactulose results were not included in the analysis. The percent recoveries of the administered sugars and the lactulose/rhamnose ratio are presented in Table 5, for which no intergroup differences were observed. Significant decreases in the recoveries of 3-O-methyl-D- glucose, D-xylose, and L-rhamnose were revealed in both examined groups.

\section{DISCUSSION}

The most important finding of this study is the lack of a significant difference in gut permeability in the patients anaesthetized either under epidural and general anaesthesia or solely under general anaesthesia.

Intraoperative abdominal aortic clamping is accompanied by many side effects involving various vital organs, such as hemodynamic disturbances with cardiac output drop and acute heart failure, decreased kidney blood perfusion and acute kidney injury, and acute spinal cord ischemia with persistent or transient neurological deficits [10]. Another organ that may suffer from hypoperfusion due to aortic clamping is the intestine [11]. Its integrity guarantees a proper barrier between the sterile peritoneal cavity and the intestinal contents that have been physiologically colonized by microorganisms. The loss of gut wall integrity may be the consequence of decreased splanchnic blood flow. Many studies have shown that this pathology may lead to endotoxemia and sepsis with serious clinical consequences, including multiorgan failure and death [12-14].

In the present study, gut permeability was assessed according to the absorption and urinary excretion of four saccharides, including 3-O-methyl-D-glucose, D-xylose, Lrhamnose and lactulose. These molecules undergo active carrier-mediated, passive carrier-mediated, transcellular and paracellular transport, respectively, in the small intestine. They are widely used as diagnostic tools to detect disturbances in these processes $[15,16]$. It is believed that the most representative parameter of intestinal wall integrity is the $L / R$ ratio, which is normally below 0.05 [17].

In the examined groups, traces of 3-O-methyl-D-glucose, $\mathrm{D}$-xylose, and L-rhamnose were detected in the urine samples collected during the first three hours of anaesthesia administration in 3 individuals from group $E \& G$ and 3 from group G. No subjects had detectable lactulose in their urine. In a study by Malagon and colleagues [17], gut permeability was assessed using a similar method in children undergoing cardiac surgery with or without cardiopulmonary bypass. All of these sugars were detected in urine samples collected during the first three hours of the procedures; however, their concentrations were low. This difference may be explained by variations in the time required for gastric emptying in small children compared with older adults, especially when the adults suffer from comorbidities that influence gastric motility, such as diabetes and/or arteriosclerosis. At the $12^{\text {th }}$ and $24^{\text {th }}$ hour after sugar administration, they could be detected in the urine, but there were no significant differences in their levels between the groups. Minimally significant decreases in the 3-O-methyl-D-glucose, D-xylose, and 
L-rhamnose concentrations were revealed at subsequent time points.

The obtained results did not confirm our hypothesis that epidural block may have beneficial effects on gut permeability disturbances induced by aortic clamping during abdominal aortic aneurysm repair. This hypothesis originated from a previous study stating that epidural block has favorable effects on intra- and postoperative intestinal function. These effects may be due to its influence on splanchnic blood flow, peristalsis improvement or intestinal shrinkage facilitated by surgery. Animal studies have indicated that epidural blocks increase mucosal blood flow in the intestines [18]. Additionally, the use of epidural block in animals with increased gut permeability has beneficial effects on gut function and splanchnic blood flow [19].

The potential beneficial effects of epidural block may have been attenuated by other factors that negatively influenced the gut barrier in our patients. The most probable influential factor was the higher rate of hypotension in group $E \& G$, which was defined as a systolic blood pressure of below $90 \mathrm{~mm} \mathrm{Hg}$ lasting for at least 15 minutes. Sympathetic block, which is an integral part of epidural block, and hypotensioninduced hypoperfusion may lead to paradoxical side effects. This observation has been confirmed by other authors [20, 21]. Another unfavorable factor is the use of catecholamines, especially in patients with advanced atherosclerosis of the intestinal arteries, which is highly probable in individuals with aortic aneurysm. In group E\&G, the rate of catecholamine infusion was relatively high (22.9\%) although there were no significant differences compared with group $G$. However, the only catecholamine used was dopamine in the dose range of 5 to $10 \mu \mathrm{g} \mathrm{kg}^{-1} \mathrm{~min}^{-1}$. Those doses should not produce substantial vasoconstriction, but this effect cannot be definitively excluded in the examined patients. Another reason for the surprising results may have been the intestinal positioning during surgery. In group $E \& G$, the intestines were more frequently (but not significantly more frequently) taken out of the peritoneal cavity and covered by a drape. Lau et al. [22] have revealed that the surgical approach (extraperitoneal versus transperitoneal) used during elective aortic aneurysm surgery affects intestinal permeability and portal endotoxemia.

There are some limitations to the present study. The main limitation is that it was not conducted in a blind fashion. In our opinion, it was not ethically justifiable to place an epidural catheter in the examined patients in the control group and administer normal saline as a placebo. This opinion is in accordance with that of Cyna and Tan, who recently published an article in Anaesthesia [23].

In conclusion, aortic clamping during open abdominal aortic repair led to unfavorable changes in intestinal perme- ability. Epidural block did not attenuate this deterioration, which may have been caused by the induced decrease in arterial pressure.

\section{ACKNOWLEDGEMENTS}

1. Source of founding: This study was financed by Polish Ministry of Science and Higher Education (grant number N N 403 287334)

2. The authors declare no conflict of interest.

\section{References:}

1. Ellard L, Djaiani G: Anaesthesia for vascular emergencies. Anaesthesia 2013; 68 (Suppl 1): 72-83. doi: 10.1111/anae.12048.

2. Jakimowicz T, Rowiński O, Gałazka Z, Solonynko B, Szmidt J: Endovascular repair of traumatic thoracic aortic rupture: a single centre experience. Kardiol Pol 2013; 71: 1273-1278. doi: 10.5603/KP.a2013.0150.

3. Karpel E, Wach E, Marszołek P, Czechowski M: Open versus percutaneous repair of abdominal aortic aneurysm. Anaesthesiol Intensive Ther 2009; 41: 120-124.

4. GierekD, Cyzowski T, Kaczmarska A, Janowska-RodakA, Budziarz B, Koczur $T$ : Perioperative prognostic factors in patients with ruptured abdominal aortic aneurysms treated in the intensive care unit. Anaesthesiol Intensive Ther 2013; 45: 25-29. doi: 10.5603/AIT.2013.0005.

5. Krzych $t$, Wybraniec M, Chudek J, Bochenek A: Perioperative management of cardiac surgery patients who are at the risk of acute kidney injury. Anaesthesiol Intensive Ther 2013; 45: 155-163. doi: 10.5603/ AIT.2013.0033.

6. Foulds $S$, Mireskandari $M$, Kalu $P$ et al.: Visceral ischemia and neutrophil activation in sepsis and organ dysfunction. J Surg Res 1998; 75: 170-176.

7. Misiołek H, Cettler M, Woroń J, Wordliczek J, Dobrogowski J, MayznerZawadzka E: The 2014 guidelines for post-operative pain management. Anaesthesiol Intensive Ther 2014; 46: 221-244. doi: 10.5603/ AIT.2014.0041.

8. Freise H, Van Aken HK: Risks and benefits of thoracic epidural anaesthesia. Br J Anaesth 2011; 107: 859-868. doi: 10.1093/bja/aer339.

9. Fujii T, Seki T, Maruoka M et al.: Lactulose-L-rhamnose intestinal permeability test in patients with liver cirrhosis. Hepatol Res 2001; 19: 158-169.

10. Levin A:The cardiovascular effects of aortic clamping and unclamping. S Afr J Anaesthesiol Analg 2010; 16: 62-71.

11. Welborn MB, Seeger JM: Prevention and management of sigmoid and pelvic ischemia associated with aortic surgery. Semin Vasc Surg 2001; 14: 255-265.

12. Kosugi S, Morisaki H, Satoh $T$ et al.: Epidural analgesia prevents endotoxin-induced gut mucosal injury in rabbits. Anesth Analg 2005; 101: 256-272.

13. Mythen MG: Postoperative gastrointestinal tract dysfunction. Anesth Analg 2005; 100: 196-204.

14. McGinley J, Lynch L, Hubbard K, McCoy D, Cunningham AJ: Dopexamine hydrochloride does not modify hemodynamic response or tissue oxygenation or gut permeability during abdominal aortic surgery. Can J Anesth 2001; 48: 238-44.

15. Marchbank T, Davison G, Oakes JR et al.: The nutriceutical bovine colostrum truncates the increase in gut permeability caused by heavy exercise in athletes. Am J Physiol Gastrointest Liver Physiol 2011; 300: G477-84. doi: 10.1152/ajpgi.00281.2010.

16. Mujagic Z, Ludidi S, Keszthelyi $D$ et al.: Small intestinal permeability is increased in diarrhoea predominant IBS, while alterations in gastroduodenal permeability in all IBS subtypes are largely attributable to confounders. Aliment Pharmacol Ther 2014; 40: 288-297. doi: 10.1111/apt.12829.

17. Malagon I, Onkenhout W, Klok G, van derPoel PF, Bovill JG, HazekampMG: Gut permeability in paediatric cardiac surgery. Br J Anaesth 2004; 94: 181-185. 10.1093/bja/aei014.

18. Sielenkämper AW, Eicker K, Van Aken H: Thoracic epidural anesthesia increases mucosal perfusion in ileum of rats. Anesthesiology 2000; 93: 844-851.

19. Daudel F, Freise $H$, Westphal $M$ et al.: Continuous thoracic epidural anesthesia improves gut mucosal microcirculation in rats with sepsis. Shock 2007; 28: 610-614. 
20. Gould TH, Grace K, Thorne G, Thomas M: Effect of thoracic epidural anaesthesia on colonic blood flow. Br J Anaesth 2002; 89: 446-451.

21. Lundberg J, Lundberg D, Norgren L, Ribbe E, Thorne J, Werner O: Intestinal haemodynamics during laparotomy: effects of thoracic epidural anaesthesia and dopamine in humans. Anesth Analg 1990; 71: 9-15.

22. Lau LL, Halliday MI, Lee B, Hannon RJ, Gardiner KR, Soong CV: Intestinal manipulation during elective aortic aneurysm surgery leads to portal endotoxaemia and mucosal barrier dysfunction. Eur J Vasc Endovasc Surg 2000; 619-624.

23. Cyna AM, Tan SG: Invasive placebo revisited. Anaesthesia 2014; 69 : 941-944. doi: 10.1111/anae.12777.

\section{Corresponding author:}

Prof. Radosław Owczuk, MD, PhD

Department of Anaesthesiology and Intensive Therapy

Medical University of Gdańsk

Smoluchowskiego 17, 80-214 Gdańsk, Poland

e-mail:r.owczuk@gumed.edu.pl

Received: 24.11.2015

Accepted: 28.02.2016 